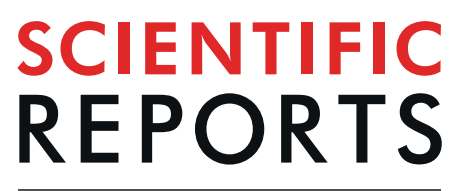

natureresearch

\title{
Bruch's membrane opening enlargement and its implication on the myopic optic nerve head
}

\begin{abstract}
Mi Sun Sung, Min Young Heo, Hwan Heo \& Sang Woo Park*
We examined the association between Bruch's membrane opening (BMO) area and various ocular parameters and investigated the implication of BMO enlargement on the myopic optic nerve head. One hundred eighty-five myopic eyes were included in this cross-sectional study. Among the included eyes, 53 having axial lengths between 26 and $27 \mathrm{~mm}$ were further analyzed to investigate the association between $\mathrm{BMO}$ area and various ocular parameters. BMO area, BMO-minimum rim width (BMO-MRW), peripapillary choroidal thickness ( $\mathrm{PCT}$ ), width of $\beta$-parapapillary atrophy with and without Bruch's membrane (PPA ${ }_{+\mathrm{BM}}$ and PPA ${ }_{-\mathrm{BM}}$ ), and presence of lamina cribrosa (LC) defect were evaluated. We found that BMO area tended to increase with increasing axial length, but varied among the highly myopic eyes even though they had similar degrees of myopia. In the subgroup analysis of eyes with axial lengths between 26 and $27 \mathrm{~mm}$, BMO area was highly variable and it significantly correlated with $\mathrm{PPA}_{- \text {вм }}$ width and temporal-inferior, nasal-inferior, and nasal BMO-MRW and $\mathrm{pCT}$. LC defects were more common in myopic eyes with enlarged BMO. A multivariate regression model revealed that higher intraocular pressure, enlarged BMO, and thinner BMO-MRW were associated with LC defects in highly myopic eyes. These findings should be considered when evaluating myopic eyes.
\end{abstract}

Histologically, the optic nerve head $(\mathrm{ONH})$ is a three-layered opening through which the axons of the retinal ganglion cells (RGCs) pass; the innermost layer is Bruch's membrane opening (BMO), the middle layer is the choroidal opening, and the third layer is the scleral canal opening ${ }^{1-3}$. Among the three openings, BMO is a clearly identifiable anatomical structure on spectral-domain optical coherence tomography (SD-OCT) and is thought to remain stable over time; thus, BMO is considered an anatomically more accurate and reliable landmark than is the conventional clinical disc margin when evaluating glaucoma ${ }^{4,5}$. Recently, BMO-based minimum rim width (MRW) measurement has become a new standard for documenting neuroretinal rim dimensions. Previous studies have reported that it has higher diagnostic accuracy for glaucoma and stronger relationship with visual field (VF) damage than do the conventional rim parameters ${ }^{6-8}$.

Myopic eyes demonstrate various morphological changes in the $\mathrm{ONH}^{9,10}$. Since the majority of structural diagnostic tools used to detect glaucoma are based on $\mathrm{ONH}$ evaluation, glaucoma diagnosis is difficult in myopic individuals with ONH deformation. In these patients, BMO-based parameters (e.g., BMO-MRW or BMO-based circumpapillary retinal nerve fiber layer [RNFL] thickness) have been proposed as good alternative diagnostic options ${ }^{11-13}$. Lee et al. ${ }^{11}$ suggested that BMO-based RNFL analysis is particularly advantageous in myopic eyes with a tilted optic disc. However, the clinical relevance of BMO-based parameters in myopic eyes is questionable, because the effect of axial elongation on BMO is not fully understood.

There are conflicting results on the association between BMO area and myopia. Some studies reported a positive correlation between BMO area and axial length ${ }^{14,15}$. In contrast, a study on a normal Caucasian population reported that $\mathrm{BMO}$ area was not significantly associated with axial length ${ }^{16}$. Moreover, in a recent prospective and longitudinal observational study, Kim et al. ${ }^{17}$ showed that the distance between two BMO points on horizontal OCT B-scan images remained stable during myopia progression. Therefore, the purpose of this study were to determine whether BMO area is affected by axial elongation and to examine the association between BMO area and various ocular parameters in myopic eyes. Additionally, we tried to elucidate the implication of BMO enlargement on the myopic ONH. 


\section{Methods}

Subjects. Study subjects were recruited from the Young Myopia Study of Chonnam National University Hospital, which is an ongoing cross-sectional study that commenced in January 2018. The present study was approved by the Institutional Review Board of Chonnam National University Hospital and it adhered to the tenets of the Declaration of Helsinki. All patients provided written informed consents prior to enrollment in the study.

The Young Myopia Study enrolled consecutive participants who visited the general eye clinic for medical check-up and met all of the inclusion criteria and none of the exclusion criteria. All subjects underwent comprehensive ophthalmologic examination consisting of the measurement of best-corrected visual acuity (BCVA), intraocular pressure (IOP) by Goldmann applanation tonometry, and refractive error by automated refraction. Anterior chamber angle assessment using gonioscopy was performed on all eyes. ONH and RNFL examination using color stereoscopic disc photography and red-free RNFL fundus photography, and Swedish Interactive Threshold Algorithm standard 30-2 perimetry with a Humphrey Field Analyzer (Carl Zeiss Meditec Inc., Dublin, CA, USA) were performed. Axial length, central corneal thickness, and corneal curvature were measured using optical low-coherence reflectometry (Lenstar; Haag-Streit AG, Koeniz, Switzerland). For all subjects, a detailed medical history was recorded.

The following inclusion criteria were used: (1) healthy subjects aged between 20 and 35 years, (2) a spherical equivalent (SE) refractive error between -0.5 and -12 diopters (D), (3) a cylinder correction of -2.0 to +2.0 $\mathrm{D}$, (4) BCVA $\geq 20 / 25$, (5) IOP $\leq 21 \mathrm{mmHg}$, (6) normal anterior chamber angles, (7) nonglaucomatous ONHs on disc photographs (an intact neuroretinal rim without peripapillary hemorrhage, thinning, or localized pallor), (8) absence of any RNFL abnormalities on red-free fundus photographs, and (9) normal VF (defined as a glaucoma hemifield test within normal limits and a pattern standard deviation within $95 \%$ confidence-interval limits) results in both eyes. We excluded subjects older than 35 years in this study, since the lenticular changes can affect refractive error, and aging itself may increase the incidence of glaucoma. Eyes having an enlarged blind spot associated with a large area of parapapillary atrophy (PPA) were also included in this study.

Subjects were excluded if they had any of the following: (1) a family history of glaucoma in a first-degree relative, (2) history of intraocular or refractive surgery, (3) pathologic myopia (patch chorioretinal atrophy, lacquer crack lesions, intrachoroidal cavitations, or choroidal neovascularization), (4) other evidence of retinal pathology, (5) opaque media, or (6) poor-quality OCT images because of irregular tear film or poor cooperation. Eligibility was determined by 2 glaucoma specialists (S.W.P and M.S.S), who evaluated optic disc appearance on stereoscopic disc photographs and RNFL defects on red-free fundus photographs. The evaluators were masked to the subject's clinical status and ocular data, and an eye was excluded from study analyses if a consensus could not be reached. When both eyes of a patient were eligible for the study, 1 eye was randomly selected.

Spectral-domain optical coherence tomography imaging. All participants underwent OCT imaging using SD-OCT (Heidelberg Spectralis SD-OCT; Spectralis software version 6.9.4; Heidelberg Engineering $\mathrm{GmbH}$, Heidelberg, Germany). One experienced operator (M.Y.H) performed all OCT scans. Magnification error was corrected using the formula provided by the manufacturer on the basis of the results of keratometry and focus setting during image acquisition. Scan images with insufficient quality (typically truncated B-scans and quality score $<30$ ) were excluded.

$\mathrm{BMO}$ and BMO-MRW were measured using the Glaucoma Module Premium Edition (GMPE) software (Fig. 1A). The scan protocol composed of 24 equally spaced radial B-scans, each with $768 \mathrm{~A}$-scans covering a $15^{\circ}$ region centered on the optic disc. Twenty-five B-scans were averaged automatically for each scan location. The software automatically segmented the internal limiting membrane (ILM) and the 48 BMO points from the 24 radial scans. All of the B-scans were manually checked, and BMO points were corrected when necessary. When $\mathrm{BMO}$ points were indiscernible, the points were fitted with a spline to derive a closed curve and a smooth contour line based on BMO points of adjacent B-scans. If BMO points were indiscernible on 4 or more consecutive B-scan images, the eyes were excluded from the study. First, BMO points were checked by one experienced evaluator (M.S.S) and then reassessed and confirmed by a senior glaucoma specialist (S.W.P). In case of any discrepancy, the eyes were excluded from the analysis. BMO area was computed automatically by the built-in software. In this scan mode, the foveal pit and 2 BMO points in each of the 2 radial B-scans that were perpendicular to each other were automatically segmented to estimate the center of BMO and determine the FoBMO axis (the line connecting the center of the fovea and BMO center), which served as a reference for the scans. The FoBMO angle was defined as the angle between the FoBMO axis and the horizontal axis of the acquired image frame.

Additionally, the GMPE software measured the distance between the termination of Bruch's membrane and the nearest point on the ILM in each B-scan image. The global and the 6 Garway-Heath regional BMO-MRW val-

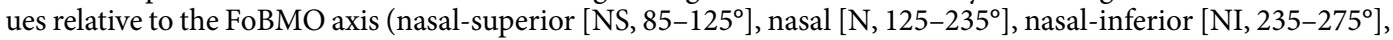
temporal-inferior [TI, $\left.275-315^{\circ}\right]$, temporal $\left[\mathrm{T}, 315-45^{\circ}\right]$, and temporal-superior $\left[\mathrm{TS}, 45-85^{\circ}\right]$ ) were calculated.

Optic nerve head measurements. The Heidelberg Spectralis OCT enhanced depth imaging (EDI) mode was used for the other $\mathrm{ONH}$ measurements (Fig. 1B). The $\mathrm{ONH}$ was scanned by centering a $15^{\circ} \times 10^{\circ}$ rectangular scan on the ONH. Each OCT volume consisted of 49 serial horizontal B-scans (4.5-mm-long lines; 50 images averaged) spaced at approximately $63-\mu \mathrm{m}$ intervals. Infrared (IR) fundus images were acquired simultaneously by using a confocal scanning laser ophthalmoscope. Three sections that passed through the ONH in the center, midsuperior, and midinferior regions were selected, and all the study parameters were measured in each of these frames by 2 independent examiners (M.S.S and H.H), in a masked fashion.

Temporal $\beta$-zone PPA margin and disc margin were defined using IR fundus images. Temporal BMO points were identified in the horizontal EDI scan image. PPA width was measured using the synchronous viewing of the IR fundus image and the selected location on the OCT scan. $\beta$-PPA width was defined as the distance between the beginning of the retinal pigment epithelium (RPE) (i.e., temporal $\beta$-PPA margin) and temporal disc margin 

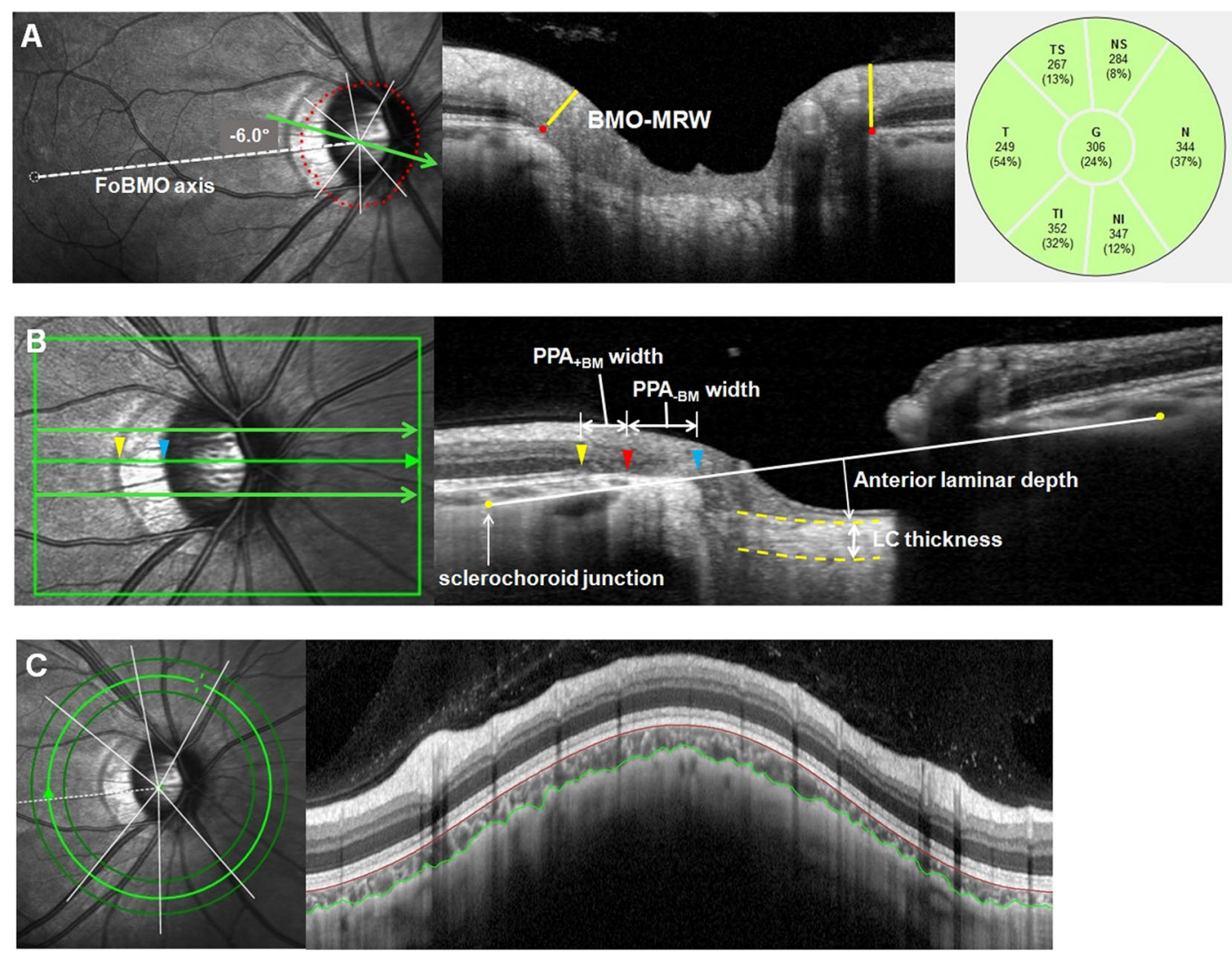

Figure 1. Methods used for ONH analysis by using SD-OCT. (A) Bruch's membrane opening (red dots) is identified on confocal scanning laser ophthalmoscopy and B-scan images. FoBMO axis (the line connecting the center of the fovea and BMO center) and FoBMO angle are also illustrated on the fundus image. The distance between the termination of BM and the nearest point on the ILM in each B-scan image is defined as BMO-MRW. The global and the 6 Garway-Heath regional BMO-MRW values (NS, N, NI, TI, T, and TS) are calculated. (B) EDI OCT scan image passing through the ONH (center, midsuperior, and midinferior) is presented. The yellow arrow head indicates the RPE termination, red arrow head indicates the termination of $\mathrm{BM}$, and blue arrow head indicates the optic disc margin. The distance from the edge of BM (red arrow head) to the margin of RPE termination (yellow arrow head) and to the optic disc margin (blue arrow head) are defined as PPA $_{+\mathrm{BM}}$ width and $\mathrm{PPA}_{-\mathrm{BM}}$ width, respectively. Anterior laminar depth is defined as the distance between the sclerochoroid junction reference line (white solid line) and the anterior border of the LC (yellow dotted line). LC thickness is defined as the distance between the anterior and posterior borders (yellow dotted lines) of the LC. (C) Circular peripapillary scan of $4.1 \mathrm{~mm}$ is obtained. After the manual segmentation of BM and the sclerochoroidal border (green solid line), peripapillary choroidal thickness was calculated. BM $=$ Bruch's membrane; $\mathrm{BMO}=$ Bruch's membrane opening; $\mathrm{MRW}=$ minimum rim width; $\mathrm{RPE}=$ retinal pigment epithelium; $\mathrm{PPA}_{+\mathrm{BM}}=\beta$-parapapillary atrophy with $\mathrm{BM} ; \mathrm{PPA}_{-\mathrm{BM}}=\beta$-parapapillary atrophy without $\mathrm{BM}$; $\mathrm{LC}=$ lamina cribrosa.

on each horizontal B-scan image. On the basis of the location of BM termination, the $\beta$-PPA was further divided into $\mathrm{PPA}_{+\mathrm{BM}}$ and $\mathrm{PPA}_{-\mathrm{BM}}\left(\gamma\right.$-zone PPA); $\mathrm{PPA}_{+\mathrm{BM}}$ width was defined as the distance from the beginning of the $\mathrm{RPE}$ to $\mathrm{BM}$, and $\mathrm{PPA}_{-\mathrm{BM}}$ was defined as the distance from the temporal disc margin to the beginning of BM. The measurement was performed using a previously described method ${ }^{18-21}$.

To overcome the effect of choroidal thickness on lamina cribrosa (LC) depth measurements, the sclerochoroid junction reference plane was used ${ }^{22,23}$. The sclerochoroid junction reference line was defined as the line connecting 2 points of the anterior scleral surface located at $1750 \mu \mathrm{m}$ from the center of BMO in each B-scan image. The vertical distance between the reference line and the anterior LC surface was measured at the center of the ONH and defined as anterior laminar depth. The anterior and posterior margins of the highly reflective region at the $\mathrm{ONH}$ vertical center in each B-scan image were used as the borders of the LC, and the perpendicular distance between these 2 borders was defined as LC thickness. In cases where the central retinal vessel trunk prevented visualization, measurements were performed on the temporal side.

The measurement was performed using a built-in caliper tool of the intrinsic OCT viewer, and average data of 3 horizontal B-scan images (center, midsuperior, and midinferior) were calculated and used in this study. The mean of the values obtained by the 2 examiners was used in the final analysis. 
Detection of lamina cribrosa defect. The EDI OCT images of the ONH were reviewed carefully for detecting focal LC defects. A focal LC defect was defined as a loss of high reflectivity from the anterior-to-posterior border of the full-thickness LC on B-scan images. To avoid false-positives, the defects were required to have had a maximal diameter greater than $100 \mu \mathrm{m}$ and a depth greater than $30 \mu \mathrm{m}$, and to have been present in two adjacent B-scans ${ }^{24,25}$. Shadows were differentiated from LC defects on the basis of their characteristic signal void behind the vessels and tissues. LC defect margins were independent of the location of the vessels and neural tissues. Example of LC defect is provided in Supplementary Fig. S1. Images were reviewed by 2 experienced glaucoma specialists (M.S.S and S.W.P), and disagreements were addressed via discussion between the 2 evaluators to achieve consensus. An eye was excluded from study analyses if a consensus could not be reached.

Choroid thickness measurement. We obtained $360^{\circ}$ circular RNFL measurements centered on BMO center. Among the 3 circular scans along the peripapillary circles (diameters of 3.5, 4.1, and $4.7 \mathrm{~mm}$ ), the 4.1-mm-diameter scans were analyzed to minimize the interference of a large PPA on the OCT scan path. For the measurement of peripapillary choroidal thickness (pCT), the upper and lower segmentation lines of the circular scan were manually delineated. The lines were adjusted to align with the inner scleral wall and posterior border of the RPE to define the outer and inner boundaries of the choroid, respectively (Fig. 1C). pCT was automatically computed using the RNFL thickness sector algorithms (global, NS, N, NI, TI, T, and TS). Subfoveal choroidal thickness was manually assessed on the EDI-OCT scan images running through the fovea. The vertical distance from the outer edge of hyper-reflective RPE to the inner border of the sclera at the fovea was measured. Images in which the RPE and inner border of the sclera could not be clearly identified were excluded. The average of data from 2 independent evaluators (M.S.S and H.H) was used in the analysis.

Statistical analysis. SPSS version 21.0 (SPSS, Chicago, IL, USA) and R version 3.3.1 were used for all statistical analyses. Agreement on $\mathrm{PPA}_{+\mathrm{BM}}$ width, PPA ${ }_{-\mathrm{BM}}$ width, LC thickness, anterior laminar depth, and peripapillary and subfoveal choroidal thickness between 2 observers was assessed using the Bland-Altman method, which plots the means against differences ${ }^{26}$. The limits of agreement were defined as the mean differences of 2 measurements \pm 1.96 standard deviation of the difference. The normality of distribution was verified using the Shapiro-Wilk normality test. Levene's test was used to check the homogeneity of variance between the different axial length groups. The main parameters were presented as the counts and proportions or mean \pm standard deviation values. Groups were compared using the chi-square test, Student's $t$ test, or Mann-Whitney $U$ test as appropriate. Linear regressions were used to search for associations between $\mathrm{BMO}$ area and various ocular parameters. Further assessment of the association between BMO area and ocular parameters was performed after including age, sex, and axial length as covariates. Coefficients with $95 \%$ confidence intervals were presented. $P$ values were adjusted to control the false discovery rate using the Benjamini-Hochberg procedure ${ }^{27}$. Factors associated with the presence of LC defects were assessed using multivariate logistic regression analysis. Confounders were identified using a threshold of $P<0.10$ in the comparison of baseline characteristics. Then, all variables with a significance level of less than 0.10 were included in the multivariate model (enter method). Sectorial BMO-MRW values were not included in the multivariate analysis to reduce collinearity in the final model. A $P$ value $<0.05$ was considered statistically significant.

\section{Results}

The study initially enrolled 205 eyes with myopia. Of these 8 eyes (3.90\%) were excluded because of poor OCT image quality. Twelve eyes (5.85\%) were additionally excluded because of indiscernible BMO on the 4 or more consecutive B-scan images or discrepancy in BMO determination between the glaucoma specialists (M.S.S and S.W.P). None of the subjects was excluded because of the disagreement on LC defect between specialists or inability to determine RPE and chorioscleral interface. Finally, 185 eyes of 205 myopic eyes were included in the analysis. Interobserver agreement, determined using Bland-Altman plots, in the measurements of $\mathrm{PPA}_{+\mathrm{BM}}$ width, PPA $_{- \text {BM }}$ width, LC thickness, and peripapillary and subfoveal choroidal thickness for all the subjects showed no systematic differences in measurements (data not shown).

The cohort included 125 male (67.57\%) and 60 female (32.43\%) subjects with a mean age of $27.06 \pm 2.77$ years. The mean SE refractive error was $-5.07 \pm 2.90 \mathrm{D}$, mean axial length was $25.91 \pm 1.37 \mathrm{~mm}$, and BMO area was $2.54 \pm 0.65 \mathrm{~mm}^{2}$. BMO area tended to increase with increasing axial length $(r=0.258, P<0.001)$ (Fig. 2). When the eyes were divided according to their axial length into 6 groups (group $\mathrm{A}=22 \mathrm{~mm} \leq$ axial length $<24 \mathrm{~mm}, \mathrm{n}=12$; group $\mathrm{B}=24 \mathrm{~mm} \leq$ axial length $<25 \mathrm{~mm}, \mathrm{n}=40$; group $\mathrm{C}=25 \mathrm{~mm} \leq$ axial length $<26 \mathrm{~mm}$, $\mathrm{n}=44$; group $\mathrm{D}=26 \mathrm{~mm} \leq$ axial length $<27 \mathrm{~mm}, \mathrm{n}=53$; group $\mathrm{E}=27 \mathrm{~mm} \leq$ axial length $<28 \mathrm{~mm}, \mathrm{n}=22$; and group $\mathrm{F}=28 \mathrm{~mm} \leq$ axial length $<30 \mathrm{~mm}, \mathrm{n}=14$ ), mean $\mathrm{SE}$ refractive error was $-0.17 \pm 0.78 \mathrm{D},-3.02 \pm 1.52 \mathrm{D}$, $-4.57 \pm 1.91 \mathrm{D},-5.62 \pm 1.74 \mathrm{D},-7.85 \pm 1.56 \mathrm{D}$, and $-10.37 \pm 1.67 \mathrm{D}$ in group $\mathrm{A}, \mathrm{B}, \mathrm{C}, \mathrm{D}, \mathrm{E}$, and F, respectively. When the homogeneity of variance of BMO area was assessed using Levene's test, a significant difference in variance was observed between the groups $(P=0.027)$. The distribution of $\mathrm{BMO}$ area across the different axial length groups is illustrated as a box-and-whisker plot in Fig. 3. Generally, a larger standard deviation of BMO area values was noted in group D.

We next focused on how the $\mathrm{ONH}$ is affected by BMO enlargement in myopic eyes. Eyes in group D were chosen for further association analysis because the variability of BMO area values was generally more pronounced in that group. We thought that the effect of BMO enlargement on $\mathrm{ONH}$ characteristics might be observed more prominently in the group with high BMO area variability. In addition, by employing strict inclusion criteria for axial length, the influence of axial elongation could be minimized. Because axial elongation itself also could accompany various morphological changes of ONH. Eventually, 53 eyes having axial lengths between 26 and $27 \mathrm{~mm}$ were further analyzed for investigating the association between BMO area and various ocular parameters. A summary of the demographic variables and the results of the OCT measurements are shown in Table 1. 


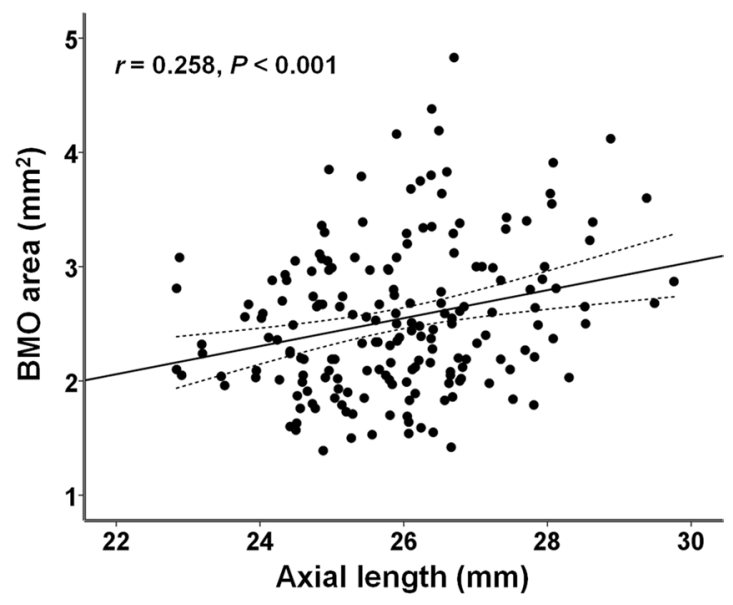

Figure 2. Distribution of BMO area values with axial length in 185 myopic subjects. The scatter plot shows that $\mathrm{BMO}$ area increases with increasing axial length. $\mathrm{BMO}=$ Bruch's membrane opening.

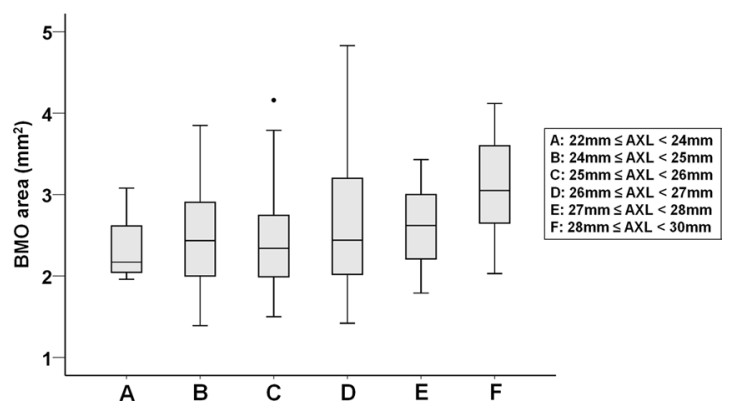

Figure 3. Box-and-whisker plots showing the distribution of $\mathrm{BMO}$ area according to the axial length group among 185 myopic eyes. $\mathrm{BMO}=$ Bruch's membrane opening; $\mathrm{AXL}=$ axial length.

Fifty-three eyes had a mean axial length of $26.43 \pm 0.27 \mathrm{~mm}$, and BMO area varied widely with a standard deviation of $0.80 \mathrm{~mm}^{2}$ (range, $1.42 \mathrm{~mm}^{2}$ to $4.83 \mathrm{~mm}^{2}$ ).

A larger BMO area was significantly associated with a larger PPA ${ }_{-B M}$ width $(P<0.001)$, deeper anterior laminar depth $(P=0.031)$, and thinner global BMO-MRW $(P<0.001)$ and global pCT $(P=0.034)$. These associations remained significant even after adjusting for age, sex, and axial length (Table 2). Sectorially, the relationship between the BMO-MRW and BMO area varied notably, with a strong correlation in the temporal-inferior, nasal-inferior, and nasal BMO-MRW sectors (all $P<0.001$, after adjusting for age, sex, and axial length) and a modest correlation in the temporal-superior and nasal-superior BMO-MRW sectors $(P=0.019$ and $P=0.024$, respectively, after adjusting for age, sex, and axial length). No significant association was found in the temporal BMO-MRW sector. The association between the corresponding sectorial pCT and BMO area showed a similar tendency as the results of the association between the sectorial BMO-MRW values and BMO area. BMO area correlated significantly with the temporal-inferior, nasal-inferior, and nasal peripapillary choroidal thicknesses ( $P=0.017, P=0.013$, and $P=0.038$, respectively, after adjusting for age, sex, and axial length). The associations between the sectorial BMO-MRW and pCT and BMO area are schematically illustrated in Fig. 4.

In this study, LC defects were identified in $20(37.74 \%)$ of the 53 myopic eyes. Notably, we found that the presence of LC defects was significantly associated with a larger BMO area $(P=0.001)$. Table 3 describes the features of the eyes with LC defects, compared with the eyes without LC defects. Eyes with LC defects had a higher IOP $(P=0.006)$, larger BMO area $(P=0.003)$, larger PPA - BM width $(P=0.004)$, deeper anterior laminar depth $(P=0.020)$, and thinner global and temporal-inferior, nasal-inferior, and nasal BMO-MRW $(P=0.003, P=0.003$, $P=0.005$, and $P=0.003$, respectively) than did eyes without LC defects. Multivariate logistic regression analysis was conducted to identify factors associated with LC defects in myopic eyes with axial lengths between 26 and $27 \mathrm{~mm}$. In order to avoid issues of multicollinearity, BMO area, PPA - BM width, and global BMO-MRW were included in the multivariable model separately, since they significantly associated with one another and showed high variance inflation factors. In the multivariate logistic regression analysis, higher IOP, larger BMO area, and thinner global BMO-MRW were associated with the presence of LC defects in myopic eyes (Table 4). 


\begin{tabular}{|c|c|}
\hline Variables & Description \\
\hline Number, $\mathrm{n}$ & 53 \\
\hline Age (years) & $26.72 \pm 2.41$ \\
\hline Male, n (\%) & $35(66.04)$ \\
\hline SE refractive error $(D)$ & $-5.62 \pm 1.74$ \\
\hline Axial length $(\mathrm{mm})$ & $26.43 \pm 0.27$ \\
\hline Central corneal thickness $(\mu \mathrm{m})$ & $552.89 \pm 27.64$ \\
\hline Corneal curvature (D) & $42.57 \pm 1.06$ \\
\hline $\mathrm{IOP}(\mathrm{mmHg})$ & $13.98 \pm 2.57$ \\
\hline $\mathrm{BMO}$ area $\left(\mathrm{mm}^{2}\right)$ & $2.59 \pm 0.80$ \\
\hline $\mathrm{PPA}_{+\mathrm{BM}}$ width $(\mu \mathrm{m})$ & $177.28 \pm 179.62$ \\
\hline $\mathrm{PPA}_{-\mathrm{BM}}(\gamma$ zone PPA $)$ width $(\mu \mathrm{m})$ & $369.43 \pm 253.34$ \\
\hline LC thickness $(\mu \mathrm{m})$ & $216.70 \pm 38.78$ \\
\hline Anterior laminar depth $(\mu \mathrm{m})$ & $330.75 \pm 108.76$ \\
\hline FoBMO angle $\left({ }^{\circ}\right)$ & $9.86 \pm 4.46$ \\
\hline Presence of LC defect, n (\%) & $20(37.74)$ \\
\hline \multicolumn{2}{|l|}{ BMO-MRW $(\mu \mathrm{m})$} \\
\hline Global & $337.58 \pm 44.85$ \\
\hline Temporal-superior & $338.87 \pm 51.40$ \\
\hline Temporal & $242.01 \pm 33.76$ \\
\hline Temporal-inferior & $355.72 \pm 55.54$ \\
\hline Nasal-inferior & $389.67 \pm 53.21$ \\
\hline Nasal & $371.51 \pm 62.13$ \\
\hline Nasal-superior & $387.58 \pm 79.95$ \\
\hline \multicolumn{2}{|l|}{ Peripapillary choroidal thickness $(\mu \mathrm{m})$} \\
\hline Global & $146.88 \pm 46.34$ \\
\hline Temporal-superior & $157.08 \pm 56.78$ \\
\hline Temporal & $135.92 \pm 52.75$ \\
\hline Temporal-inferior & $120.65 \pm 42.75$ \\
\hline Nasal-inferior & $125.69 \pm 43.55$ \\
\hline Nasal & $161.40 \pm 49.98$ \\
\hline Nasal-superior & $168.98 \pm 56.14$ \\
\hline Subfoveal choroidal thickness $(\mu \mathrm{m})$ & $250.45 \pm 83.02$ \\
\hline
\end{tabular}

Table 1. Summary of subjects $(26 \mathrm{~mm} \leq$ axial length $<27 \mathrm{~mm})$ characteristics. $\mathrm{SE}=$ spherical equivalent; $\mathrm{D}=$ diopter; $\mathrm{IOP}=$ intraocular pressure; $\mathrm{BMO}=$ Bruch's membrane opening; $\mathrm{PPA}_{+\mathrm{BM}}=\beta$-parapapillary atrophy with Bruch's membrane; $\mathrm{PPA}_{-\mathrm{BM}}=\beta$-parapapillary atrophy without Bruch's membrane; $\mathrm{LC}=$ lamina cribrosa; FoBMO angle = angle of fovea-to-BMO-center axis relative to the horizontal axis of the image frame; $\mathrm{MRW}=$ minimum rim width. Data are mean \pm standard deviation unless otherwise indicated.

\section{Discussion}

Recent studies have suggested that BMO would be a good anatomical landmark for assessing ONH in myopic eyes, and BMO-MRW measurement based on the BMO has emerged as a promising option in diagnosing myopic glaucoma ${ }^{11-13,28}$. However, how $\mathrm{BMO}$ is affected by axial elongation has been determined in less detail, and to the best of our knowledge, no other studies have thoroughly investigated the implication of BMO enlargement on the myopic ONH. In this study, we found that BMO area tended to increase with axial elongation. However the correlation between $\mathrm{BMO}$ area and axial length was relatively weak. Interestingly, we identified a notable variation in $\mathrm{BMO}$ area among otherwise healthy myopic eyes, and this suggested that BMO enlargement does not necessarily occur in all myopic eyes. BMO enlargement was associated with PPA ${ }_{-B M}$ width, BMO-MRW, and pCT thinning at the temporal-inferior, nasal-inferior, and nasal sectors, as well as the presence of LC defects. The current study findings are important because they indicate that physicians should be aware of sectorial BMO-MRW thinning in myopic eyes with large BMO when evaluating patients with glaucoma, and it might help better understand the pathogenesis of myopic ONH deformations.

Jonas et al. ${ }^{29}$ described the myopia-associated widening of BMO around the $\mathrm{ONH}$ in their histomorphometric study. The studies performed on normal Japanese and Australian populations reported a significant positive correlation between BMO area and axial length ${ }^{14,30}$. Nakanishi et al. ${ }^{15}$ also showed that BMO area was significantly associated with axial length. Our findings are consistent with those of previous reports, and indicate that BMO might undergo morphological changes during axial elongation. Whereas, another study on a normal Caucasian population demonstrated that BMO area was unrelated to axial length. However, that study might not be compared to ours because of the different inclusion criteria; they included only eyes with SE refractive error within $-6 \mathrm{D}$ and excluded highly myopic eyes ${ }^{16}$. In a recent prospective longitudinal evaluation of childhood myopia, Kim et al. ${ }^{17}$ found that BMO remained stable during myopia progression. However, their observation period was 


\begin{tabular}{|c|c|c|c|c|}
\hline \multirow[b]{2}{*}{ Variables } & \multicolumn{2}{|l|}{ Univariable } & \multicolumn{2}{|c|}{ Adjusted for age, sex and axial length } \\
\hline & Coefficient $(95 \% \mathrm{CI})$ & $P$-value ${ }^{*}$ & Coefficient $(95 \% \mathrm{CI})$ & $P$-value ${ }^{*}$ \\
\hline Age (years) & $-0.004(-0.090,0.083)$ & 0.933 & - & - \\
\hline Female gender & $-0.007(-0.043,0.072)$ & 0.570 & - & - \\
\hline Axial length $(\mathrm{mm})$ & $0.252(-0.583,1.088)$ & 0.547 & - & - \\
\hline Central corneal thickness $(\mu \mathrm{m})$ & $-0.013(-0.018,0.002)$ & 0.112 & $-0.010(-0.018,0.003)$ & 0.135 \\
\hline Corneal curvature (D) & $-0.037(-0.267,0.194)$ & 0.749 & $-0.041(-0.274,0.192)$ & 0.725 \\
\hline IOP (mmHg) & $0.049(-0.038,0.136)$ & 0.262 & $0.058(-0.033,0.148)$ & 0.208 \\
\hline $\mathrm{PPA}_{+\mathrm{BM}}$ width $(\mu \mathrm{m})$ & $-0.000081(-0.001,0.001)$ & 0.896 & $-0.000085(-0.001,0.001)$ & 0.892 \\
\hline $\mathrm{PPA}_{-\mathrm{BM}}(\gamma$ zone PPA $)$ width $(\mu \mathrm{m})$ & $0.002(0.001,0.002)$ & $<0.001$ & $0.002(0.001,0.002)$ & $<0.001$ \\
\hline LC thickness $(\mu \mathrm{m})$ & $-0.000283(-0.006,0.005)$ & 0.922 & $-0.001(-0.007,0.005)$ & 0.835 \\
\hline Anterior laminar depth $(\mu \mathrm{m})$ & $0.002(0.001,0.004)$ & 0.031 & $0.003(0.001,0.005)$ & 0.016 \\
\hline FoBMO angle $\left(^{\circ}\right)$ & $0.039(-0.026,0.104)$ & 0.232 & $0.037(-0.030,0.104)$ & 0.274 \\
\hline LC defect & $0.723(0.314,1.132)$ & 0.001 & $0.720(0.307,1.132)$ & 0.001 \\
\hline \multicolumn{5}{|l|}{ BMO-MRW $(\mu \mathrm{m})$} \\
\hline Global & $-0.008(-0.013,-0.004)$ & $<0.001$ & $-0.008(-0.013,-0.004)$ & $<0.001$ \\
\hline Temporal-superior & $-0.005(-0.009,-0.001)$ & 0.019 & $-0.005(-0.009,-0.001)$ & 0.019 \\
\hline Temporal & $-0.004(-0.011,0.002)$ & 0.172 & $-0.004(-0.011,0.002)$ & 0.176 \\
\hline Temporal-inferior & $-0.007(-0.010,-0.003)$ & $<0.001$ & $-0.007(-0.010,-0.003)$ & $<0.001$ \\
\hline Nasal-inferior & $-0.008(-0.012,-0.005)$ & $<0.001$ & $-0.008(-0.012,-0.005)$ & $<0.001$ \\
\hline Nasal & $-0.006(-0.009,-0.003)$ & $<0.001$ & $-0.006(-0.009,-0.003)$ & $<0.001$ \\
\hline Nasal-superior & $-0.003(-0.006,0.001)$ & 0.025 & $-0.003(-0.006,-0.0004)$ & 0.024 \\
\hline \multicolumn{5}{|c|}{ Peripapillary choroidal thickness $(\mu \mathrm{m})$} \\
\hline Global & $-0.005(-0.010,-0.0003)$ & 0.034 & $-0.005(-0.010,-0.0002)$ & 0.040 \\
\hline Temporal-superior & $-0.003(-0.007,0.001)$ & 0.185 & $-0.003(-0.006,0.001)$ & 0.208 \\
\hline Temporal & $-0.004(-0.008,0.000)$ & 0.077 & $-0.004(-0.008,0.001)$ & 0.093 \\
\hline Temporal-inferior & $-0.006(-0.011,-0.001)$ & 0.016 & $-0.006(-0.011 .-0.001)$ & 0.017 \\
\hline Nasal-inferior & $-0.006(-0.011,-0.002)$ & 0.010 & $-0.006(-0.011,-0.001)$ & 0.013 \\
\hline Nasal & $-0.005(-0.009,-0.0003)$ & 0.034 & $-0.005(-0.009,-0.0002)$ & 0.038 \\
\hline Nasal-superior & $-0.003(-0.007,0.001)$ & 0.134 & $-0.003(-0.007,0.001)$ & 0.141 \\
\hline Subfoveal choroidal thickness $(\mu \mathrm{m})$ & $-0.002(-0.004,0.001)$ & 0.169 & $-0.002(-0.004,0.001)$ & 0.204 \\
\hline
\end{tabular}

Table 2. Associations between the BMO area and various clinical and ocular parameters among the 53 subjects $(26 \mathrm{~mm} \leq$ axial length $<27 \mathrm{~mm}) . \mathrm{CI}=$ confidence interval; $\mathrm{D}=$ diopter; $\mathrm{IOP}=$ intraocular pressure; $\mathrm{BMO}=$ Bruch's membrane opening; $\mathrm{PPA}_{+\mathrm{BM}}=\beta$-parapapillary atrophy with Bruch's membrane; PPA $\mathrm{Bм}=\beta$-parapapillary atrophy without Bruch's membrane; $\mathrm{LC}=$ lamina cribrosa; FoBMO angle $=$ angle of foveato-BMO-center axis relative to the horizontal axis of the image frame; MRW $=$ minimum rim width. Factors with statistical significance are shown in boldface. ${ }^{*} P$ values were adjusted with Benjamini-Hochberg procedure.

relatively short (2 years). Additionally, a careful assessment of their result showed a tendency for BMO distance to increase as time progressed, even though it did not reach the level of statistical significance $(P=0.100)$.

Previous studies have shown that BM thickness and length in the macular lesion were not affected by axial elongation $^{31,32}$. It has been suggested that the axial elongation-related increase in optic disc-fovea distance was the result of the development and enlargement of the parapapillary gamma zone (in other words, $\mathrm{PPA}_{-\mathrm{BM}}$ in this study) and that macular BM neither expands nor gets thinner ${ }^{31,32}$. It might be due to the non elastic and relatively high biomechanical strength of BM. Thus, the mechanical strain within the BM during axial elongation might cause the enlargement of $\mathrm{BMO}$, that is the physiological hole, instead of $\mathrm{BM}$ stretching.

In this study, a negative correlation was observed between BMO-MRW and BMO area. The dependency of BMO-MRW on BMO size is well known ${ }^{6,14,15}$. With a larger BMO area, the axons of RGCs spread over an increased perimeter, and therefore, the measured BMO-MRW seems to be diminished while still possessing the same number of axons. Interestingly, we found that the association between BMO-MRW and BMO area differed sectorially, with a strong correlation in the temporal-inferior, nasal-inferior, and nasal sectors and a modest correlation in the temporal-superior and nasal-superior BMO-MRW sectors. No correlation was found in the temporal sector. Our results might indicate that BMO enlargement occurs asymmetrically, mainly at the inferior and nasal lesions in myopic eyes. Although the statistical significance was weak, the significant correlations between $\mathrm{pCT}$ and $\mathrm{BMO}$ area at the corresponding sectors might support our speculation. Therefore, we hypothesize that the temporal site of BMO remains constant, and the enlargement occurs more predominantly at the inferior and nasal sectors of the BMO during axial elongation. Future research that measures BM thickness around the $\mathrm{ONH}$ might help understand $\mathrm{BMO}$ changes during myopia progression.

In myopic eyes with axial lengths between 26 and $27 \mathrm{~mm}, \mathrm{pCT}$ was thinnest at the temporal-inferior sector. Our finding agrees with those of previous reports ${ }^{33,34}$. Gupta et al. ${ }^{33}$ explored the profile of the peripapillary choroid and found that it was thinnest inferiorly in both myopic and emmetropic eyes. Importantly, the temporal-inferior peripapillary choroid became significantly thinner as BMO enlarged in this study. Given that 


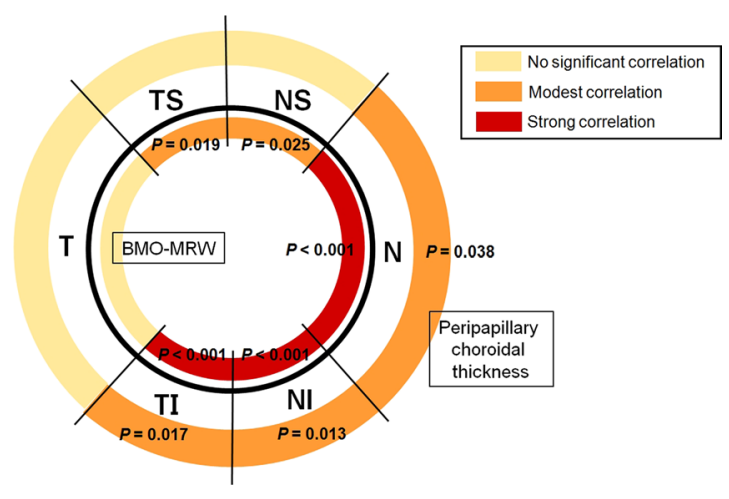

Figure 4. Diagram illustrating the association between BMO-MRW (inner color-coded circle) and peripapillary choroidal thickness (outer color-coded circle) and BMO area in 53 myopic eyes with axial length between 26 and $27 \mathrm{~mm}$. $P$ values shown are adjusted for age, sex, and axial length. $B M O=$ Bruch's membrane opening; $\mathrm{MRW}=$ minimum rim width; $\mathrm{NS}=$ nasal-superior; $\mathrm{N}=$ nasal; $\mathrm{NI}=$ nasal-inferior; $\mathrm{TI}=$ temporalinferior; $\mathrm{T}=$ temporal; $\mathrm{TS}=$ temporal-superior.

\begin{tabular}{|c|c|c|c|}
\hline Variables & $\begin{array}{l}\text { Eyes with LC defect } \\
(\mathbf{n}=20)\end{array}$ & $\begin{array}{l}\text { Eyes without LC defect } \\
(\mathbf{n}=33)\end{array}$ & $P$-value \\
\hline Age (years) & $26.23 \pm 1.24$ & $27.00 \pm 2.86$ & 0.272 \\
\hline Male, n (\%) & $14(70.00)$ & $21(63.64)$ & 0.768 \\
\hline SE refractive error (D) & $-5.96 \pm 1.51$ & $-5.41 \pm 1.85$ & 0.271 \\
\hline Axial length (mm) & $26.44 \pm 0.25$ & $26.42 \pm 0.28$ & 0.812 \\
\hline Central corneal thickness $(\mu \mathrm{m})$ & $548.75 \pm 28.93$ & $555.39 \pm 26.97$ & 0.402 \\
\hline Corneal curvature (D) & $42.59 \pm 1.13$ & $42.56 \pm 1.03$ & 0.928 \\
\hline IOP (mmHg) & $15.50 \pm 2.45$ & $13.39 \pm 2.50$ & 0.006 \\
\hline $\mathrm{BMO}$ area $\left(\mathrm{mm}^{2}\right)$ & $3.04 \pm 0.86$ & $2.31 \pm 0.62$ & 0.003 \\
\hline $\mathrm{PPA}_{+\mathrm{BM}}$ width $(\mu \mathrm{m})$ & $213.00 \pm 182.56$ & $155.64 \pm 177.12$ & 0.264 \\
\hline $\mathrm{PPA}_{-\mathrm{BM}}(\gamma$ zone PPA $)$ width $(\mu \mathrm{m})$ & $507.95 \pm 286.20$ & $285.48 \pm 190.80$ & 0.004 \\
\hline LC thickness $(\mu \mathrm{m})$ & $216.95 \pm 48.06$ & $216.55 \pm 32.74$ & 0.971 \\
\hline Anterior laminar depth $(\mu \mathrm{m})$ & $380.70 \pm 131.23$ & $300.48 \pm 80.57$ & 0.020 \\
\hline FoBMO angle $\left({ }^{\circ}\right)$ & $-5.14 \pm 3.23$ & $-5.65 \pm 3.50$ & 0.598 \\
\hline \multicolumn{4}{|l|}{ BMO-MRW $(\mu \mathrm{m})$} \\
\hline Global & $316.65 \pm 34.15$ & $350.27 \pm 46.22$ & 0.003 \\
\hline Temporal-superior & $324.05 \pm 37.50$ & $347.85 \pm 56.91$ & 0.103 \\
\hline Temporal & $235.05 \pm 28.21$ & $246.24 \pm 36.48$ & 0.246 \\
\hline Temporal-inferior & $327.80 \pm 56.08$ & $372.64 \pm 48.61$ & 0.003 \\
\hline Nasal-inferior & $364.00 \pm 49.90$ & $405.21 \pm 49.62$ & 0.005 \\
\hline Nasal & $340.30 \pm 54.11$ & $390.42 \pm 59.64$ & 0.003 \\
\hline Nasal-superior & $368.30 \pm 66.52$ & $399.27 \pm 85.94$ & 0.174 \\
\hline \multicolumn{4}{|l|}{ Peripapillary choroidal thickness $(\mu \mathrm{m})$} \\
\hline Global & $137.79 \pm 41.02$ & $152.12 \pm 48.98$ & 0.287 \\
\hline Temporal-superior & $146.74 \pm 51.91$ & $163.03 \pm 59.35$ & 0.324 \\
\hline Temporal & $123.47 \pm 37.08$ & $143.09 \pm 59.30$ & 0.149 \\
\hline Temporal-inferior & $112.32 \pm 32.50$ & $125.45 \pm 47.47$ & 0.290 \\
\hline Nasal-inferior & $118.95 \pm 40.81$ & $129.58 \pm 45.20$ & 0.402 \\
\hline Nasal & $155.00 \pm 51.84$ & $165.09 \pm 49.31$ & 0.489 \\
\hline Nasal-superior & $157.95 \pm 56.48$ & $175.33 \pm 55.81$ & 0.287 \\
\hline Subfoveal choroidal thickness $(\mu \mathrm{m})$ & $233.85 \pm 65.31$ & $260.52 \pm 91.60$ & 0.223 \\
\hline
\end{tabular}

Table 3. Comparison between eyes with or without lamina cribrosa defect. $\mathrm{D}=$ diopter; $\mathrm{IOP}=$ intraocular pressure; $\mathrm{BMO}=$ Bruch's membrane opening; $\mathrm{PPA}_{+\mathrm{BM}}=\beta$-parapapillary atrophy with Bruch's membrane; $\mathrm{PPA}_{-\mathrm{BM}}=\beta$-parapapillary atrophy without Bruch's membrane; $\mathrm{LC}=$ lamina cribrosa; FoBMO angle $=$ angle of fovea-to-BMO-center axis relative to the horizontal axis of the image frame; $\mathrm{MRW}=$ minimum rim width. Data are mean \pm standard deviation unless otherwise indicated. Factors with statistical significance are shown in boldface. 


\begin{tabular}{|c|c|c|c|c|c|c|c|c|}
\hline \multirow[b]{2}{*}{ Variables } & \multicolumn{2}{|l|}{ Univariate } & \multicolumn{2}{|c|}{ Multivariate (Model 1) } & \multicolumn{2}{|c|}{ Multivariate (Model 2) } & \multicolumn{2}{|c|}{ Multivariate (Model 3) } \\
\hline & $\begin{array}{l}\text { Odds Ratio } \\
\text { (95\% CI) }\end{array}$ & $P$-value & $\begin{array}{l}\text { Odds Ratio } \\
(95 \% \text { CI })\end{array}$ & $P$-value & $\begin{array}{l}\text { Odds Ratio } \\
(95 \% \mathrm{CI})\end{array}$ & $P$-value & $\begin{array}{l}\text { Odds Ratio } \\
(95 \% \text { CI })\end{array}$ & $P$-value \\
\hline IOP & $1.406(1.075,1.839)$ & 0.013 & $1.315(1.006,1.719)$ & 0.045 & $1.301(1.004,1.699)$ & 0.046 & $1.506(1.123,2.020)$ & 0.006 \\
\hline Anterior laminar depth & $1.008(1.001,1.015)$ & 0.019 & $1.005(0.997,1.014)$ & 0.184 & $1.004(0.997,1.012)$ & 0.264 & $1.004(0.996,1.011)$ & 0.314 \\
\hline $\mathrm{BMO}$ area & $3.752(1.548,9.090)$ & 0.003 & $2.728(1.119,6.653)$ & 0.017 & & & & \\
\hline PPA $_{- \text {BM }}$ width & $1.004(1.001,1.007)$ & 0.006 & & & $1.003(0.999,1.006)$ & 0.163 & & \\
\hline Global BMO-MRW & $0.978(0.961,0.995)$ & 0.012 & & & & & $0.973(0.953,0.993)$ & 0.010 \\
\hline
\end{tabular}

Table 4. Factors associating with lamina cribrosa defect in highly myopic eyes ( $26 \mathrm{~mm} \leq$ axial length $<27 \mathrm{~mm})$. $\mathrm{CI}=$ confidence interval; $\mathrm{IOP}=$ intraocular pressure; $\mathrm{BMO}=$ Bruch's membrane opening; $\mathrm{PPA}_{-\mathrm{BM}}=\beta$-parapapillary atrophy without Bruch's membrane; $\mathrm{MRW}=$ minimum rim width. Factors with statistical significance are shown in boldface. $P$ values were adjusted with Benjamini-Hochberg procedure.

vascular support for the prelaminar portion of the optic disc originates from the peripapillary choroid ${ }^{35}$, choroi- $^{-}$ dal thinning might be linked to vascular insufficiency, contributing to the increased glaucoma susceptibility in the temporal-inferior region. However, it remains unknown whether the development of myopic glaucoma in the temporal-inferior region is more prevalent in eyes with enlarged BMO than in eyes without enlarged BMO. Future longitudinal studies would be needed to clarify this question.

Because BMO size is not consistent among individuals and shows large between-subject variability in the normal population ${ }^{14,16}$, one may argue that the large BMO found in this study might not be the result of axial elongation-associated $\mathrm{ONH}$ deformation, and might just be an inborn characteristic. A limitation of the current study is that our findings are the result of cross-sectional comparisons among myopic eyes, and we did not observe the eyes longitudinally. However, we found a significant positive correlation between $\mathrm{PPA}_{-\mathrm{BM}}$ width and $\mathrm{BMO}$ area after adjusting for age, sex, and axial length. Previous clinical and histomorphometric studies have revealed that $\mathrm{PPA}_{-\mathrm{BM}}$ (sometimes referred to as gamma zone PPA) width increased greatly with increasing axial length, and PPA - BM is thought to be the result of scleral stretching associated with eyeball elongation ${ }^{36,37}$. Therefore, we suggest that the eyes with large BMO described in our study might have undergone more mechanical strain on the optic disc during axial elongation, and longer PPA ${ }_{-B M}$ width might be an indirect evidence of BMO enlargement.

The LC of the ONH has been proposed as the primary site for glaucoma pathogenesis ${ }^{38}$. Among the various morphological changes found in patients with glaucoma, posterior displacement of the LC is considered a key feature of glaucomatous optic nerve damage. Few studies have conducted detailed investigations of LC depth in healthy myopic eyes ${ }^{39}$. In a study performed on a Korean population, Yun et al. ${ }^{39}$ compared LC depth in different axial length subgroups and reported that LC depth did not differ significantly in normal eyes. However, they used $\mathrm{BMO}$ as a reference plane, and did not consider the morphological changes of the myopic ONH. In the current study, we measured LC depth relative to the sclerochoroid junction to eliminate the influence of choroidal thickness and found that anterior laminar depth was significantly associated with BMO area. Our finding might indicate that BMO enlargement also accompanies the backward movement of the LC.

LC defects have been extensively studied with respect to their potential as a marker for progressive damage to RGCs in myopic glaucoma ${ }^{25,40-42}$. Han et al.$^{40}$ previously described that, even among subjects without glaucoma, LC defects were found in $27.8 \%$ of myopic eyes. As in their reports, the current study also found LC defects in highly myopic eyes without any clinical sign of glaucoma and that LC defects were relatively common (37.74\%). Notably, BMO area was an important parameter in LC defect formation. We propose that mechanical strain on the LC during BMO enlargement might cause LC defects.

Additionally, IOP was significantly associated with LC defects in highly myopic eyes. In our earlier study, we demonstrated a significant association between IOP and optic disc rotation ${ }^{42}$. In that study, we hypothesized that IOP may result in additional stress on the posterior sclera during axial elongation and might therefore affect the development of myopic ONH deformation. Similarly, IOP-related stress within the connective tissues of the LC might play a role in LC defects. Since the level of IOP-related stress for a given level of IOP is principally determined by the geometry of the load-bearing tissues of the $\mathrm{ONH}^{43}$, our hypothesis does not seem unreasonable, even though all subjects in this study had normal IOP.

The strength of this study is the inclusion of young, healthy subjects with high myopia and no ocular disease, who were thus free of confounding factors. However, our study also had some limitations. First, we excluded eyes if BMO was not clearly detectable on 4 or more consecutive B-scan images. There is a possibility of exclusion of potential study subjects that might have influenced the results of our study; thus, some of the parameters may be underestimated. In a recent study, Zheng et al. ${ }^{44}$ reported that $28.0 \%$ of highly myopic eyes showed indiscernible $\mathrm{BMO}$ commonly over the superotemporal and inferotemporal meridians. We also found that a significant proportion of highly myopic eyes had indiscernible BMO over at least 1 meridian. Nevertheless, to minimize the exclusion of potential highly myopic subjects, the exclusion criteria were relaxed in this study, under the assumption that BMO is not a tortuous line but a closed curve with a smooth contour. As a result, only about $6 \%$ of the eyes were excluded. Therefore, the effect that the excluded subjects might have had would be negligible. Second, the cause-and-effect relationship between BMO enlargement and significantly associated factors cannot be ascertained owing to the cross-sectional nature of our study. Third, because our study subjects were Korean, the current findings may not be generalized to other ethnic populations. Fourth, we did not evaluate the location and number of LC defects. Future analyses using more detailed information of LC defects may provide additional insights. 
In conclusion, BMO area varied among the highly myopic eyes having similar axial lengths. Although our data do not present the underlying mechanism, BMO seems to be enlarged more prominently at the inferior and nasal sectors. Clinicians should be aware of BMO-MRW thinning at the temporal-inferior, nasal-inferior, and nasal sectors when evaluating myopic eyes. Additionally, we clearly observed that the highly myopic eyes with enlarged BMO exhibited characteristic morphologic features of peripapillary choroidal thinning, posterior displacement of the LC, and LC defects. Considering their significant association with glaucoma, our results are important because they highlight the importance of $\mathrm{BMO}$ in myopic eyes. Nevertheless, the role of BMO enlargement in the pathogenesis of myopic glaucoma and its value as a predictor of future damage remains to be determined.

\section{Data availability}

The datasets generated during the current study are available from the corresponding author on reasonable request.

Received: 16 August 2019; Accepted: 4 December 2019;

Published online: 20 December 2019

\section{References}

1. Strouthidis, N. G., Yang, H., Downs, J. C. \& Burgoyne, C. F. Comparison of clinical and three-dimensional histomorphometric optic disc margin anatomy. Invest Ophthalmol Vis Sci. 50, 2165-2174 (2009).

2. Jonas, J. B. \& Xu, L. Histological changes of high axial myopia. Eye (Lond). 28, 113-117 (2014).

3. Jonas, J. B., Holbach, L. \& Panda-Jonas, S. Peripapillary ring: histology and correlations. Acta Ophthalmol. 92, e273-e279 (2014).

4. Chauhan, B. C. \& Burgoyne, C. F. From clinical examination of the optic disc to clinical assessment of the optic nerve head: a paradigm change. Am J Ophthalmol. 156, 218-227 (2013).

5. Belghith, A. et al. Does the location of Bruch's membrane opening change over time? Longitudinal analysis using San Diego Automated Layer Segmentation Algorithm (SALSA). Invest Ophthalmol Vis Sci. 57, 675-682 (2016).

6. Chauhan, B. C. et al. Enhanced detection of open-angle glaucoma with an anatomically accurate optical coherence tomographyderived neuroretinal rim parameter. Ophthalmology. 120, 535-543 (2013).

7. Pollet-Villard, F., Chiquet, C., Romanet, J. P., Noel, C. \& Aptel, F. Structure-function relationships with spectral-domain optical coherence tomography retinal nerve fiber layer and optic nerve head measurements. Invest Ophthalmol Vis Sci. 55, 2953-2962 (2014).

8. Gardiner, S. K. et al. A method to estimate the amount of neuroretinal rim tissue in glaucoma: comparison with current methods for measuring rim area. Am J Ophthalmol. 157, 540-549 (2014).

9. Morgan, I. G., Ohno-Matsui, K. \& Saw, S. M. Myopia. Lancet. 379, 1739-1748 (2012).

10. Jonas, J. B., Gusek, G. C. \& Naumann, G. O. Optic disk morphometry in high myopia. Graefes Arch Clin Exp Ophthalmol. 226, 587-590 (1998)

11. Lee, E. J., Lee, K. M., Kim, H. \& Kim, T. W. Glaucoma diagnostic ability of the new circumpapillary retinal nerve fiber layer thickness analysis based on Bruch's membrane opening. Invest Ophthalmol Vis Sci. 57, 4194-4204 (2016).

12. Sastre-Ibañez, M. et al. Utility of Bruch membrane opening-based optic nerve head parameters in myopic subjects. Eur J Ophthalmol. 28, 42-46 (2018).

13. Rebolleda, G., Casado, A., Oblanca, N. \& Muñoz-Negrete, F. J. The new Bruch's membrane opening - minimum rim width classification improves optical coherence tomography specificity in tilted discs. Clin Ophthalmol. 10, 2417-2425 (2016).

14. Araie, M. et al. Determinants and characteristics of Bruch's membrane opening and Bruch's membrane opening-minimum rim width in a normal Japanese population. Invest Ophthalmol Vis Sci. 58, 4106-4113 (2017).

15. Nakanishi, H. et al. Association of Bruch's membrane opening and optic disc morphology to axial length and visual field defects in eyes with primary open-angle glaucoma. Graefes Arch Clin Exp Ophthalmol. 256, 599-610 (2018).

16. Chauhan, B. C. et al. Bruch's membrane opening minimum rim width and retinal nerve fiber layer thickness in a normal White population: A multicenter study. Ophthalmology. 122, 1786-1794 (2015).

17. Kim, M., Choung, H. K., Lee, K. M., Oh, S. \& Kim, S. H. Longitudinal changes of optic nerve head and peripapillary structure during childhood myopia progression on OCT: Boramae Myopia Cohort Study report 1. Ophthalmology. 125, 1215-1223 (2018).

18. Kim, Y. W., Lee, E. J., Kim, T. W., Kim, M. \& Kim, H. Microstructure of $\beta$-zone parapapillary atrophy and rate of retinal nerve fiber layer thinning in primary open-angle glaucoma. Ophthalmology. 121, 1341-1349 (2014).

19. Yamada, H. et al. Microstructure of peripapillary atrophy and subsequent visual field progression in treated primary open-angle glaucoma. Ophthalmology. 123, 542-551 (2016).

20. Lee, S. H., Lee, E. J. \& Kim, T. W. Topographic correlation between juxtapapillary choroidal thickness and microstructure of parapapillary atrophy. Ophthalmology. 123, 1965-1973 (2016).

21. Sung, M. S., Heo, H. \& Park, S. W. Microstructure of parapapillary atrophy is associated with parapapillary microvasculature in myopic eyes. Am J Ophthalmol. 192, 157-168 (2018).

22. Vianna, J. R. et al. Serial Changes in lamina cribrosa depth and neuroretinal parameters in glaucoma: Impact of choroidal thickness. Ophthalmology. 124, 1392-1402 (2017).

23. Moghimi, S. et al. Lamina cribrosa and choroid features and their relationship to stage of pseudoexfoliation glaucoma. Invest Ophthalmol Vis Sci. 59, 5355-5365 (2018)

24. Kiumehr, S. et al. In vivo evaluation of focal lamina cribrosa defects in glaucoma. Arch Ophthalmol. 130, 552-559 (2012).

25. Sawada, Y., Araie, M., Ishikawa, M. \& Yoshitomi, T. Multiple temporal lamina cribrosa defects in myopic eyes with glaucoma and their association with visual field defects. Ophthalmology. 124, 1600-1611 (2017).

26. Bland, J. M. \& Altman, D. G. Statistical methods for assessing agreement between two methods of clinical measurement. Lancet. 1, 307-310 (1986)

27. Benjamini, Y. \& Hochberg, Y. Controlling the false discovery rate: a practical and powerful approach to multiple testing. J R Stat Soc B. 57, 289-300 (1995).

28. Malik, R. et al. Diagnostic accuracy of optical coherence tomography and scanning laser tomography for identifying glaucoma in myopic eyes. Ophthalmology. 123, 1181-1189 (2016).

29. Jonas, J. B., Ohno-Matsui, K., Spaide, R. F., Holbach, L. \& Panda-Jonas, S. Macular Bruch's membrane defects and axial length: association with gamma zone and delta zone in peripapillary region. Invest Ophthalmol Vis Sci. 54, 1295-1302 (2013).

30. Sanfilippo, P. G., Huynh, E., Yazar, S., Hewitt, A. W. \& Mackey, D. A. Spectral-domain optical coherence tomography-derived characteristics of Bruch membrane opening in a young adult Australian population. Am J Ophthalmol. 165, 154-163 (2016).

31. Jonas, J. B. et al. Macular Bruch's membrane length and axial length. The Beijing Eye Study. PLoS One. 10, e0136833 (2015).

32. Bai, H. X. et al. Bruch's membrane thickness in relationship to axial length. PLoS One. 12, e0182080 (2017).

33. Gupta, P. et al. Peripapillary choroidal thickness in young Asians with high myopia. Invest Ophthalmol Vis Sci. 56, 1475-1481 (2015). 
34. Yamashita, T. et al. Correlations between local peripapillary choroidal thickness and axial length, optic disc tilt, and papillo-macular position in young healthy eyes. PLoS One. 12, e0186453 (2017).

35. Hayreh, S. S. Blood supply of the optic nerve head and its role in optic atrophy, glaucoma, and oedema of the optic disc. $\mathrm{Br} J$ Ophthalmol. 53, 721-748 (1969).

36. Jonas, J. B. et al. Parapapillary atrophy: histological gamma zone and delta zone. PLoS One. 7, e47237 (2012).

37. Dai, Y., Jonas, J. B., Huang, H., Wang, M. \& Sun, X. Microstructure of parapapillary atrophy: beta zone and gamma zone. Invest Ophthalmol Vis Sci. 54, 2013-2018 (2013).

38. Quigley, H. A., Addicks, E. M., Green, W. R. \& Maumenee, A. E. Optic nerve damage in human glaucoma. II. The site of injury and susceptibility to damage. Arch Ophthalmol. 99, 635-649 (1981).

39. Yun, S. C. et al. Lamina cribrosa depth according to the level of axial length in normal and glaucomatous eyes. Graefes Arch Clin Exp Ophthalmol. 253, 2247-2253 (2015).

40. Han, J. C., Cho, S. H., Sohn, D. Y. \& Kee, C. The characteristics of lamina cribrosa defects in myopic eyes with and without openangle glaucoma. Invest Ophthalmol Vis Sci. 57, 486-494 (2016).

41. Kimura, Y. et al. Lamina cribrosa defects and optic disc morphology in primary open angle glaucoma with high myopia. PLoS One. 9, el15313 (2014)

42. Sung, M. S., Kang, Y. S., Heo, H. \& Park, S. W. Characteristics of optic disc rotation in myopic eyes. Ophthalmology. 123, 400-407 (2016).

43. Bellezza, A. J., Hart, R. T. \& Burgoyne, C. F. The optic nerve head as a biomechanical structure: initial finite element modeling. Invest Ophthalmol Vis Sci. 41, 2991-3000 (2000).

44. Zheng, F., Wu, Z. \& Leung, C. K. S. Detection of Bruch's membrane opening in healthy individuals and glaucoma patients with and without high myopia. Ophthalmology. 125, 1537-1546 (2018).

\section{Acknowledgements}

This research was supported by the Bio \& Medical Technology Development Program of the NRF funded by the Korean government (NRF-2017M3A9E8023019), and by the grant of Chonnam National University Hospital Biomedical Research Institute (BCRI18019 and BCRI18025). The funding organizations had no role in the design or conduct of this research.

\section{Author contributions}

The design and conduct of the study (M.S.S., M.Y.H., H.H. and S.W.P.); collection of data (M.S.S., M.Y.H. and H.H.); management, analysis, and interpretation of data (M.S.S., H.H. and S.W.P.); and preparation of the manuscript (M.S.S. and S.W.P.). All authors reviewed the manuscript.

\section{Competing interests}

The authors declare no competing interests.

Additional information

Supplementary information is available for this paper at https://doi.org/10.1038/s41598-019-55926-w.

Correspondence and requests for materials should be addressed to S.W.P.

Reprints and permissions information is available at www.nature.com/reprints.

Publisher's note Springer Nature remains neutral with regard to jurisdictional claims in published maps and institutional affiliations.

(c) (1) Open Access This article is licensed under a Creative Commons Attribution 4.0 International License, which permits use, sharing, adaptation, distribution and reproduction in any medium or format, as long as you give appropriate credit to the original author(s) and the source, provide a link to the Creative Commons license, and indicate if changes were made. The images or other third party material in this article are included in the article's Creative Commons license, unless indicated otherwise in a credit line to the material. If material is not included in the article's Creative Commons license and your intended use is not permitted by statutory regulation or exceeds the permitted use, you will need to obtain permission directly from the copyright holder. To view a copy of this license, visit http://creativecommons.org/licenses/by/4.0/.

(C) The Author(s) 2019 